\title{
ROBUSTNESS OF INTERVAL TOEPLITZ MATRICES IN FUZZY ALGEBRA
}

\author{
Helena MYŠKOVÁ \\ Department of Mathematics and Theoretical Informatics, Faculty of Electrical Engineering and Informatics, Technical University of \\ Košice, Nemcovej 32, 04200 Košice, Slovak Republic, tel.: +421 55602 2445, e-mail: helena.myskova@tuke.sk
}

\begin{abstract}
Fuzzy algebra is an algebraic structure in which classical addition and multiplication are replaced by $\oplus$ and $\otimes$, where $a \oplus b=$ $\max \{a, b\}, a \otimes b=\min \{a, b\}$. Fuzzy discrete dynamic systems can be introduced by fuzzy matrices and are useful for describing knowledge engineering, scheduling, cluster analysis, fuzzy logic programs, diagnosis of technical devices or medical diagnosis.

The paper deals with robust matrices over fuzzy algebra. There are defined the terms of the possible and universal robustness of interval matrices. The work describes the necessary and sufficient condition for the possible and universal robustness of interval Toeplitz matrices which satisfy a certain condition, called the condition $\mathscr{C}^{*}$.
\end{abstract}

Keywords: fuzzy algebra, Toeplitz matrix, interval Toeplitz matrix, possible robustness, universal robustness

\section{INTRODUCTION}

Studying matrix properties in fuzzy algebra, where addition and multiplication are formally replaced by operations of maximum and minimum, is of great importance for applications in various areas. Fuzzy discrete dynamic systems can be introduced by fuzzy matrices and are useful for describing knowledge engineering, scheduling, cluster analysis, fuzzy logic programs [6], diagnosis of technical devices [13], [14] or medical diagnosis [12].

Periodic behaviour of fuzzy matrices with corresponding polynomial algorithms were studied in $[5]$ and [10]. However, in practice we deal often with inexact input data. This leads to demand replace scalar matrices by so-called interval matrices ( [1]]).

The main aim of this paper is to describe so called robust matrices and introduce the necessary and sufficient conditions for the possible and universal robustness of an interval Toeplitz matrices.

\section{BACKGROUND OF THE PROBLEM}

The fuzzy algebra $\mathscr{B}$ is the triple $(B, \oplus, \otimes)$, where $(B, \leq)$ is a bounded linearly ordered set with binary operations maximum and minimum, denoted by $\oplus$ and $\otimes$, respectively. The least element in $B$ will be denoted by $O$, the greatest one by $I$.

By $\mathbb{N}$ we denote the set of all natural numbers and by $\mathbb{N}_{0}$ the set $\mathbb{N}_{0}=\mathbb{N} \cup\{0\}$. The greatest common divisor of a set $S \subseteq \mathbb{N}$ is denoted by $\operatorname{gcd} S$ and the least common multiple by $1 \mathrm{~cm} S$. For a given natural number $n \in \mathbb{N}$, we use the notations $N=\{1,2, \ldots, n\}$.

For any $n \in \mathbb{N}, B(n, n)$ denotes the set of all square matrices of order $n$ and $B(n)$ denotes the set of all $n$ dimensional column vectors over $\mathscr{B}$. The matrix operations over $\mathscr{B}$ are defined formally in the same manner (with respect to $\oplus, \otimes)$ as matrix operations over any field. The $r$-th power of a matrix $A$ is denoted by $A^{r}$, with elements $\left(A^{r}\right)_{i j}$.

For $A=\left(a_{i j}\right) \in B(n, n), C=\left(c_{i j}\right) \in B(n, n)$ we write $A \leq C(A<C)$ if $a_{i j} \leq c_{i j}\left(a_{i j}<c_{i j}\right)$ holds for all $i, j \in N$.

By digraph we understand a pair $\mathscr{G}=\left(V_{\mathscr{G}}, E_{\mathscr{G}}\right)$, where $V_{\mathscr{G}}$ is a non-empty finite set, called the node set, and $E_{\mathscr{G}} \subseteq$ $V_{\mathscr{G}} \times V_{\mathscr{G}}$, called the arc set. A digraph $\mathscr{G}^{\prime}=\left(V_{\mathscr{G}^{\prime}}, E_{\mathscr{G}^{\prime}}\right)$ is a subdigraph of digraph $\mathscr{G}$, if $V_{\mathscr{G}^{\prime}} \subseteq V_{\mathscr{G}}$ and $E_{\mathscr{G}^{\prime}} \subseteq E_{\mathscr{G}}$. Specially, $\mathscr{G} / V_{\mathscr{G}}$ stands for the subdigraph induced by vertex set $V_{\mathscr{G} /}$.

A walk in a digraph $\mathscr{G}$ is the sequence of nodes and $\operatorname{arcs} \mathscr{P}=\left(v_{0}, e_{1}, v_{1}, e_{2}, v_{2}, \ldots, v_{l-1}, e_{l}, v_{l}\right)$ such that $e_{k}=\left(v_{k-1}, v_{k}\right) \in E_{\mathscr{G}}$ for $k=1,2, \ldots, l$. A walk in $\mathscr{G}$ is a trail if all its arcs are distinct. The number $l$ is the length of the trail $\mathscr{P}$ and is denoted by $\ell(\mathscr{P})$. If $v_{0}=v_{l}$, then $\mathscr{P}$ is called a cycle. A cycle is elementary if all nodes except the terminal node are distinct. A digraph is called strongly connected if any two distinct nodes of $\mathscr{G}$ are contained in a common cycle. By a strongly connected component of $\mathscr{G}$ we mean a maximal strongly connected subdigraph of $\mathscr{G}$. A strongly connected component $\mathscr{K}=\left(V_{\mathscr{K}}, E_{\mathscr{K}}\right)$ is called non-trivial if there is a cycle of positive length in $\mathscr{K}$. For any non-trivial strongly connected component $\mathscr{K}$ is the period of $\mathscr{K}$ defined as

$\operatorname{per} \mathscr{K}=\operatorname{gcd}\{\ell(c) ; c$ is a cycle in $\mathscr{K}, \ell(c)>0\}$.

If $\mathscr{K}$ is trivial, then per $\mathscr{K}=1$. By $\mathrm{SCC}^{\star} \mathscr{G}$ we denote the set of all non-trivial strongly connected components of $\mathscr{G}$.

Lemma 2.1. Let $\mathscr{K} \in \mathrm{SCC}^{\star} \mathscr{G}, \quad \mathscr{K}^{\prime} \in \mathrm{SCC}^{\star} \mathscr{G}^{\prime}$ and $\mathscr{K} \subseteq \mathscr{K}^{\prime}$. Then per $\mathscr{K}^{\prime} \mid \operatorname{per} \mathscr{K}$.

Proof. Denote by $C_{\mathscr{K}}$ and $C_{\mathscr{K}}$ the sets of all cycles in $\mathscr{K}$ and $\mathscr{K}^{\prime}$, respectively. Since

$$
\begin{gathered}
C_{\mathscr{K}}=\{\ell(c) ; c \text { is a cycle in } \mathscr{K}, \ell(c)>0\} \subseteq \\
\subseteq\left\{\ell\left(c^{\prime}\right) ; c^{\prime} \text { is a cycle in } \mathscr{K}^{\prime}, \ell\left(c^{\prime}\right)>0\right\}=C_{\mathscr{K}},
\end{gathered}
$$

we obtain

$$
\operatorname{per} \mathscr{K}^{\prime}=\operatorname{gcd} C_{\mathscr{K}^{\prime}} \mid \operatorname{gcd} C_{\mathscr{K}}=\operatorname{per} \mathscr{K} .
$$

Further, we define the period of the digraph $\mathscr{G}$ as follows

$$
\operatorname{per} \mathscr{G}=\operatorname{lcm}\left\{\operatorname{per} \mathscr{K} ; \mathscr{K} \in \mathrm{SCC}^{\star} \mathscr{G}\right\} .
$$

For a given matrix $A \in B(n, n)$ the symbol $\mathscr{G}(A)=$ $\left(V_{\mathscr{G}(A)}, E_{\mathscr{G}(A)}\right)$ stands for the complete, edge-weighted digraph associated with $A$, i.e., the vertex set of $\mathscr{G}(A)$ is $N$, and the capacity of any edge $(i, j) \in E_{\mathscr{G}(A)}$ is $a_{i j}$. In addition, for given $h \in B$, the threshold digraph $\mathscr{G}(A, h)$ is the 
digraph with the vertex set $V_{\mathscr{G}(A, h)}=N$ and the edge set $E_{\mathscr{G}(A, h)}=\left\{(i, j) ; i, j \in N, a_{i j} \geq h\right\}$.

The following lemma describes the relation between matrices and corresponding threshold digraphs and follows from the transitivity of ordering.

Lemma 2.2. [7] Let $A, C \in B(n, n)$. Let $h, h_{1}, h_{2} \in B$.

(i) If $A \leq C$, then $\mathscr{G}(A, h) \subseteq \mathscr{G}(C, h)$,

(ii) if $h_{1} \leq h_{2}$, then $\mathscr{G}\left(A, h_{2}\right) \subseteq \mathscr{G}\left(A, h_{1}\right)$.

Let $A \in B(n, n)$ and $x \in B(n)$. The orbit $\mathscr{O}(A, x)$ of $x=x^{(0)}$ generated by $A$ is the sequence

$$
x^{(0)}, x^{(1)}, x^{(2)}, \ldots, x^{(n)}, \ldots,
$$

where $x^{(r)}=A^{r} \otimes x^{(0)}$ for each $r \in \mathbb{N}$.

For a given matrix $A \in B(n, n)$, the element $\lambda \in B$ and the $n$-tuple $x \in B(n)$ are the so-called eigenvalue of $A$ and eigenvector of $A$, respectively, if

$$
A \otimes x=\lambda \otimes x
$$

The eigenspace $V(A, \lambda)$ is defined as the set of all eigenvectors of $A$ with associated eigenvalue $\lambda$, i.e.,

$$
V(A, \lambda)=\{x \in B(n) ; A \otimes x=\lambda \otimes x\} .
$$

Let $\lambda \in B$. A matrix $A \in B(n, n)$ is ultimately $\lambda$-periodic if there are natural numbers $p$ and $R$ such that the following holds:

$$
A^{k+p}=\lambda \otimes A^{k} \text { for all } k \geq R .
$$

The smallest natural number $p$ with the above property is called the period of $A$, denoted by $\operatorname{per}(A, \lambda)$. In case $\lambda=I$ let us denote $\operatorname{per}(A, I)$ by abbreviation per $A$. It is known that if a matrix is ultimately $\lambda$-periodic, then it is ultimately $I$-periodic and $\operatorname{per}(A, \lambda)=\operatorname{per}(A, I)$.

According to [5] we define

$$
\operatorname{SCC}^{\star}(A)=\cup\left\{\operatorname{SCC}^{\star} \mathscr{G}(A, h) ; h \in\left\{a_{i j} ; i, j \in N\right\}\right\}
$$

Theorem 2.1. [5] Let $A \in B(n, n)$. Then

$$
\operatorname{per} A=\operatorname{lcm}\left\{\operatorname{per} \mathscr{K} ; \mathscr{K} \in \mathrm{SCC}^{\star}(A)\right\} \text {. }
$$

Let us denote

$$
T(A, \lambda)=\{x \in B(n) ; \mathscr{O}(A, x) \cap V(A, \lambda) \neq \emptyset\} .
$$

Definition 2.1. Let $A=\left(a_{i j}\right) \in B(n, n), \lambda \in B$. A matrix $A$ is called $\lambda$-robust if $T(A, \lambda)=B(n)$.

A $\lambda$-robust matrix with $\lambda=I$ is called robust matrix. It is easy to see that if $A=\left(a_{i j}\right)$ is ultimately $\lambda$-periodic and $\lambda \geq \max _{i, j \in N} a_{i j}$, then $\lambda \geq \max _{i, j \in N} a_{i j}^{k}$ and $A^{k+p}=\lambda \otimes A^{k}=A^{k}$ for $k \geq R$. Hence the necessary condition for $A=\left(a_{i j}\right)$ to be ultimately $\lambda$-periodic is $\lambda \geq \max _{i, j \in N} a_{i j}^{k}$. The results we shall formulate for $\lambda=I$, as well as the methods used to prove them, can be generalized for arbitrary $\lambda \in\left[\max _{i, j \in N} a_{i j}, I\right]$.

We recall a result of the paper [10] adapted for $\lambda=I$.

Lemma 2.3. [10] Let $A=\left(a_{i j}\right) \in B(n, n)$. Then $A$ is robust if and only if per $A=1$.

Note that an $O\left(n^{3}\right)$ algorithm for finding per $A$ is presented in $[5]$.

\section{PERIODICITY OF TOEPLITZ MATRICES}

In this section we shall deal with the special class of matrices, the Toeplitz matrices. A Toeplitz matrix contains the same element on every diagonal, which is paralell to the main diagonal.

Definition 3.1. Let $a_{-n+1}, \ldots, a_{-1}, a_{0}, a_{1}, \ldots, a_{n-1} \in B$. A matrix $A \in B(n, n)$ of the form

$A=\left(\begin{array}{cccccc}a_{0} & a_{1} & a_{2} & \ldots & a_{n-2} & a_{n-1} \\ a_{-1} & a_{0} & a_{1} & \ldots & a_{n-3} & a_{n-2} \\ a_{-2} & a_{-1} & a_{0} & \ldots & a_{n-4} & a_{n-3} \\ \vdots & \vdots & \vdots & & \vdots & \vdots \\ a_{-n+2} & a_{-n+3} & a_{-n+4} & \ldots & a_{0} & a_{1} \\ a_{-n+1} & a_{-n+2} & a_{-n+3} & \ldots & a_{-1} & a_{0}\end{array}\right)$

is called a Toeplitz matrix. We denote a Toeplitz matrix by

$$
A=\operatorname{Tp}\left(a_{-n+1}, \ldots, a_{-1}, a_{0}, a_{1}, \ldots, a_{n-1}\right) .
$$

We denote

(i) $N^{-}=\{-n+1,-n+2, \ldots,-1\}$,

$N^{+}=\{1,2, \ldots, n-1\}$,

$N^{*}=\{-n+1,-n+2, \ldots,-1,0,1, \ldots, n-2, n-1\}$,

(ii) $h^{+}(A)=\max _{i \in N^{+}} a_{i}$,

$h^{-}(A)=\max _{i \in N^{-}} a_{i}$,

$h(A)=\min \left\{h^{+}(A), h^{-}(A)\right\}$,

(iii) $I^{+}(A)=\left\{i \in N^{+} ; a_{i} \geq h(A)\right\}$,

$I^{-}(A)=\left\{i \in N^{-} ; a_{i} \geq h(A)\right\}$,

$I(A)=\left\{i \in N^{*} ; a_{i} \geq h(A)\right\}$,

iv) $\tilde{N}(A)= \begin{cases}N^{+} & \text {if } h(A)=h^{+}(A), \\ N^{-} & \text {otherwise. }\end{cases}$

The possibility $\tilde{N}(A)=N^{+}$contains a case where $h^{+}(A)=$ $h^{-}(A)$, too.

Remark 3.1. Note that $h(A)$ is the maximal threshold level for which both sets $I^{+}(A), I^{-}(A)$ are non-empty. For any $h^{\prime}>h(A)$, the threshold digraph $\mathscr{G}\left(A, h^{\prime}\right)$ is either trivial (there is no edge in $\mathscr{G}\left(A, h^{\prime}\right)$ ) in the case $a_{0} \leq h(A)$, or all strongly connected components consist of exactly one node with the loop in the case $a_{0}>h^{\prime}$.

According to [2], we denote by $\mathscr{C}^{*}$ the following condition of a Toeplitz matrix:

$$
i-j \leq n \text { holds for every } i \in I^{+}(A), j \in I^{-}(A) .
$$

Theorem 3.1. [2]

Let $A=T p\left(a_{-n+1}, \ldots, a_{-1}, a_{0}, a_{1}, \ldots, a_{n-1}\right) \in B(n, n)$ be $a$ Toeplitz matrix fulfilling condition $\mathscr{C}^{*}$. Then per $A=\operatorname{per} \mathscr{K}$ for any component $\mathscr{K} \in \mathrm{SCC}^{\star} \mathscr{G}(A, h(A))$.

Remark 3.2. If a Toeplitz matrix $A \in B(n, n)$ fulfills condition $\mathscr{C}^{*}$, then all strongly connected components in $\mathscr{G}(A, h(A))$ are non-trivial, but not isomorphic in general. 
Theorem 3.2. [2] Let $A \in B(n, n)$ be a Toeplitz matrix fulfilling condition $\mathscr{C}^{*}$, let $I(A)=\left\{i_{0}, i_{1}, \ldots, i_{k-1}\right\}$. Then

$$
\begin{array}{r}
\operatorname{per} A=\operatorname{gcd}\left(\frac{i_{0}-i_{1}}{\operatorname{gcd}\left(n, i_{0}, i_{1}\right)}, \frac{i_{0}-i_{2}}{\operatorname{gcd}\left(n, i_{0}, i_{1}, i_{2}\right)}, \ldots\right. \\
\left.\ldots, \frac{i_{0}-i_{k-1}}{\operatorname{gcd}\left(n, i_{0}, \ldots, i_{k-1}\right)}\right) .
\end{array}
$$

Remark 3.3. According to [2], the period of Toeplitz matrix can be computed in $O(n)$ time.

\section{ROBUSTNESS OF INTERVAL MATRICES}

In this section we shall deal with matrices with interval elements. Similarly to [1], [3], [4], [8], [9], [11] we define an interval matrix A.

Definition 4.1. Let $\underline{A}, \bar{A} \in B(n, n), \underline{A} \leq \bar{A}$. An interval matrix $\mathbf{A}$ with bounds $\underline{A}$ and $\bar{A}$ is defined as follows

$$
\mathbf{A}=[\underline{A}, \bar{A}]=\{A \in B(n, n) ; \underline{A} \leq A \leq \bar{A}\} .
$$

Investigating the robustness of an interval matrix $\mathbf{A}$, the following questions can arise: Is A robust for some $A \in \mathbf{A}$ or for all $A \in \mathbf{A}$ ?

Definition 4.2. An interval matrix $\mathbf{A}$ is called

- possibly robust if there exists a matrix $A \in \mathbf{A}$ such that A is robust,

- universally robust if each matrix $A \in \mathbf{A}$ is robust.

Possible and universal robustness of general interval matrices were studied in [7].

Let us denote $H=\left\{\bar{a}_{i j} ; i, j \in N\right\} \cup\left\{\underline{a}_{i j} ; i, j \in N\right\}$.

Theorem 4.1. [7] An interval matrix $\mathbf{A}$ is possibly robust if and only if for each $h \in H$ and for each $\mathscr{K} \in \operatorname{SCC}^{\star} \mathscr{G}(\bar{A}, h)$ such that per $\mathscr{K} \neq 1$ the digraph $\mathscr{G}(\underline{A}, h) / V_{\mathscr{K}}$ is acyclic.

For a given $h \in H$ let us denote $\tilde{V}_{h}=N \backslash \bigcup_{j=1}^{s_{h}} V_{\mathscr{K}_{h}^{j}}$, where $\mathscr{K}_{h}^{1}, \ldots, \mathscr{K}_{h}^{s_{h}} \in \operatorname{SCC}^{\star} \mathscr{G}(\underline{A}, h)$.

Theorem 4.2. [7] Let $\mathbf{A}$ be an interval matrix. Then $\mathbf{A}$ is universally robust if and only if $\underline{A}$ is robust and $\operatorname{per}(\mathscr{G}(\underline{A}, h) \cup c)=1$ for each $h \in H$ and for each cycle $c \in \mathscr{G}(\overline{\bar{A}}, h) / \tilde{V}_{h}$.

According to [7], the complexity of checking the possible robustness of a given interval matrix is $O\left(n^{5}\right)$, whereby checking the universal robustness has exponentially large complexity.

\section{ROBUSTNESS OF INTERVAL TOEPLITZ MA- TRICES}

The possible and universal robustness of interval Toeplitz matrices are studied in this section. The necessary and sufficient conditions which can be checked in polynomial time are given.
Definition 5.1. Let $\underline{A}, \bar{A}$ be Toeplitz matrices of order $n$ such that $\underline{A} \leq \bar{A}$. An interval Toeplitz matrix $\mathbf{A}^{T p}$ is the set of all Toeplitz matrices $A \in[\underline{A}, \bar{A}]$. We denote an interval Toeplitz matrix $\mathbf{A}^{T p}$ by abbreviation

$$
\begin{gathered}
\mathbf{A}^{T p}=\operatorname{Tp}\left(\left[\underline{a}_{-n+1}, \bar{a}_{-n+1}\right], \ldots\left[\underline{a}_{-1}, \bar{a}_{-1}\right],\left[\underline{a}_{0}, \bar{a}_{0}\right],\right. \\
\left.\left[\underline{a}_{1}, \bar{a}_{1}\right], \ldots,\left[\underline{a}_{n-1}, \bar{a}_{n-1}\right]\right) .
\end{gathered}
$$

There are matrices in $\mathbf{A}$ that are not Toeplitz, so $\mathbf{A} \neq \mathbf{A}^{T p}$. On the other hand $\underline{A}, \bar{A} \in \mathbf{A}^{T p}$, therefore the set $\mathbf{A}^{T p}$ is always non-empty.

\subsection{Possible robustness}

Definition 5.2. We say that an interval Toeplitz matrix $\mathbf{A}^{T p}$ fulfills condition $\mathscr{C}^{*}$ if each Toeplitz matrix $A \in \mathbf{A}^{T p}$ fulfills condition $\mathscr{C}^{*}$.

Let us define the Toeplitz matrix $\tilde{A}=$ $\operatorname{Tp}\left(\tilde{a}_{-n+1}, \ldots, \tilde{a}_{-1}, \tilde{a}_{0}, \tilde{a}_{1}, \ldots, \tilde{a}_{n-1}\right)$ as follows:

$\tilde{a}_{i}= \begin{cases}\min \left\{h(\underline{A}), \bar{a}_{i}\right\} & \text { for } i \in \tilde{N}(\underline{A}), \\ \bar{a}_{i} & \text { for } i \notin \tilde{N}(\underline{A}) .\end{cases}$

It is easy to see that $h(\tilde{A})=h(\underline{A})$ and $\tilde{N}(\tilde{A})=\tilde{N}(\underline{A})$.

Lemma 5.1. Let $\mathbf{A}^{T p}$ be an interval Toeplitz matrix and $\tilde{A}$ be the matrix defined by (3). Then $I(A) \subseteq I(\tilde{A})$ for each $A \in \mathbf{A}^{T p}$.

Proof. We shall prove that $I^{+}(A) \subseteq I^{+}(\tilde{A}), I^{-}(A) \subseteq I^{-}(\tilde{A})$ and if $0 \in I(A)$, then $0 \in I(\tilde{A})$, for each $A \in \mathbf{A}^{T p}$.

Without any loss of generality we can suppose that $\tilde{N}(\underline{A})=N^{+}$. Thus $I^{+}(\tilde{A})=\left\{i \in N^{+} ; \tilde{a}_{i}=h(\tilde{A})\right\}=\{i \in$ $\left.N^{+} ; \bar{a}_{i} \geq h(\tilde{A})\right\}$. Let $A \in \mathbf{A}^{T p}$ be arbitrary. We shall distinguish two cases.

Case 1. If $\tilde{N}(A)=N^{+}$, then $I^{+}(A)=\left\{i \in N^{+} ; a_{i}=\right.$ $h(A)\}$ and $I^{-}(A)=\left\{i \in N^{-} ; a_{i} \geq h(A)\right\}$. For the sets $I^{-}(A), I^{-}(\tilde{A})$ we get

$I^{-}(A)=\left\{i \in N^{-} ; a_{i} \geq h(A)\right\} \subseteq\left\{i \in N^{-} ; \tilde{a}_{i} \geq h(\tilde{A})\right\}=I^{-}(\tilde{A})$.

Further, we will prove that $I^{+}(A) \subseteq I^{+}(\tilde{A})$. Let $r \in$ $I^{+}(A)$ be arbitrary. We get

$$
\bar{a}_{r} \geq a_{r}=\max _{k \in N^{+}} a_{k} \geq h(\tilde{A})
$$

which implies $r \in I^{+}(\tilde{A})$. Consequently $I^{+}(A) \subseteq I^{+}(\tilde{A})$.

Case 2. If $\tilde{N}(A)=N^{-}$, then

$$
\begin{gathered}
I^{-}(A)=\left\{i \in N^{-} ; a_{i}=h(A)\right\} \subseteq\left\{i \in N^{-} ; a_{i} \geq h(\tilde{A})\right\} \subseteq \\
\left\{i \in N^{-} ; \tilde{a}_{i} \geq h(\tilde{A})\right\}=I^{-}(\tilde{A}) .
\end{gathered}
$$

Further, we prove that $I^{+}(A) \subseteq I^{+}(\tilde{A})$. We obtain

$$
\begin{gathered}
I^{+}(A)=\left\{i \in N^{+} ; a_{i} \geq h(A)\right\} \subseteq\left\{i \in N^{+} ; a_{i} \geq h(\tilde{A})\right\} \subseteq \\
\left\{i \in N^{+} ; \bar{a}_{i} \geq h(\tilde{A})\right\}=I^{+}(\tilde{A}) .
\end{gathered}
$$

In both cases if $0 \in I(A)$, then $\tilde{a}_{0}=\bar{a}_{0} \geq a_{0} \geq h(A) \geq h(\tilde{A})$, so $0 \in I(\tilde{A})$.

Theorem 5.1. An interval Toeplitz matrix $\mathbf{A}^{T p}$ fulfills condition $\mathscr{C}^{*}$ if and only if the matrix $\tilde{A}$ fulfills condition $\mathscr{C}^{*}$. 
Proof. If the matrix $\tilde{A}$ fulfills condition $\mathscr{C}^{*}$, i.e., $i-j \leq n$ for each $i \in I^{+}(\tilde{A}), j \in I^{-}(\tilde{A})$, then, in view of Lemma 5.1, for each $A \in \mathbf{A}^{T p}$ the inequality $i-j \leq n$ holds for each $i \in I^{+}(A), j \in I^{-}(A)$. Thus an interval Toeplitz matrix fulfills condition $\mathscr{C}^{*}$.

The converse implication is trivial.

Theorem 5.2. An interval Toeplitz matrix $\mathbf{A}^{T p}$ fulfilling condition $\mathscr{C}^{*}$ is possibly robust if and only if the matrix $\tilde{A}$ is robust.

Proof. Since $I(A) \subseteq I(\tilde{A})$, according to 2 we get $\operatorname{per} \tilde{A} \mid \operatorname{per} A$. If $\tilde{A}$ is not robust, i.e., $\operatorname{per} \tilde{A} \neq 1$, then $\operatorname{per} A \neq 1$ for each $A \in \mathbf{A}^{T p}$. Thus an interval Toeplitz matrix $\mathbf{A}^{T p}$ is not possibly robust.

The converse implication is trivial.

According to Theorem 5.2 checking the possible robustness of a given interval Toeplitz matrix fulfilling condition $\mathscr{C}^{*}$ consists of $O(n)$ arithmetic operations needed for the construction of the matrix $\tilde{A}, O(n)$ operations for checking whether $\tilde{A}$ fulfills condition $\mathscr{C}^{*}$ and $O(n)$ operations for computing per $\tilde{A}$ by 2 . So the complexity of checking the possible robustness of a given interval Toeplitz matrix fulfilling condition $\mathscr{C}^{*}$ is $O(n)$, which substantially improves the $O\left(n^{5}\right)$ algorithm for checking the possible robustness of an interval matrix in general case.

Example 5.1. Let

$$
\mathbf{A}^{T p}=T([1,2],[4,5],[2,2],[1,2],[2,4],[3,5],[1,2]) .
$$

We decide whether $\mathbf{A}^{T p}$ fulfills condition $\mathscr{C}^{*}$ and in positive case we check the possible robustness of $\mathbf{A}^{T p}$.

First, we compute $h(\underline{A})$ and construct the matrix $\tilde{A}$. We have $h^{+}(\underline{A})=3, h^{-}(\underline{A})=4$ and $h(\underline{A})=3$, which implies $\tilde{N}(\underline{A})=N^{+}$. By $\sqrt{3}$, we get $\tilde{A}=T(2,5,2,2,3,3,2)$. We have $h(\tilde{A})=3$ and $I(\tilde{A})=\{-2,1,2\}$. Since $i-j \leq 4=n$ for each $i \in I^{+}(\tilde{A}), j \in I^{-}(\tilde{A})$, the matrix $\tilde{A}$ fulfills condition $\mathscr{C}^{*}$. According to Theorem 5.1 $\mathbf{A}^{T p}$ fulfills condition $\mathscr{C}^{*}$.

Further, we check the possible robustness of $\mathbf{A}^{T p}$. We compute per $\tilde{A}$ by 2 ):

$$
\operatorname{per} \tilde{A}=\operatorname{gcd}\left(\frac{-3}{\operatorname{gcd}(4,-2,1)}, \frac{-4}{\operatorname{gcd}(4,-2,1,2)}\right)=1,
$$

so the matrix $\tilde{A}$ is robust. In view of Theorem 5.2, the given interval Toeplitz matrix is possibly robust.

\subsection{Universal robustness}

Theorem 5.3. Let $\mathbf{A}^{T p}$ be an interval Toeplitz matrix fulfilling condition $\mathscr{C}^{*}$. If $h(\underline{A})=h(\bar{A})$ and the matrix $\underline{A}$ is robust, then $\mathbf{A}^{T p}$ is universally robust.

Proof. Let $\mathbf{A}^{T p}$ be an interval Toeplitz matrix fulfilling condition $\mathscr{C}^{*}$. Suppose that $\mathbf{A}^{T p}$ is not universally robust and the matrix $A$ is robust. If $A \in \mathbf{A}^{T p}$ is not robust, then by Theorem 3.1 we obtain per $\tilde{\mathscr{K}}=\operatorname{per} A \neq 1$ for each $\tilde{\mathscr{K}} \in \mathrm{SCC}^{\star} \mathscr{G}(A, h(A))$. In view of Remark 3.2 and Lemma 2.2. for each $h \leq h(\underline{A})$ and for each $\mathscr{K} \in \operatorname{SCC}^{\star} \mathscr{G}(A, h)$ there exists $\mathscr{K}^{\prime} \in \mathrm{SCC}^{\star} \mathscr{G}(\underline{A}, h(\underline{A}))$ such that $\mathscr{K}^{\prime} \subseteq \mathscr{K}$.
The robustness of $A$ implies that per $\mathscr{K}^{\prime}=1$ for each $\mathscr{K}^{\prime} \in \mathrm{SCC}^{\star} \mathscr{G}(\underline{A}, h(\underline{A}))$. According to Lemma 2.1 we get per $\mathscr{K}=1$ for each $\mathscr{K} \in \mathrm{SCC}^{\star} \mathscr{G}(A, h)$ and for each $h \leq$ $h(A)$. Then the existence of $\tilde{\mathscr{K}} \in \mathrm{SCC}^{\star} \mathscr{G}(A, h(A))$ such that per $\tilde{\mathscr{K}} \neq 1$ implies $h(A)>h(\underline{A})$. Hence $h(\underline{A})<h(\bar{A})$. $\square$

\section{Example 5.2. Let}

$$
\mathbf{A}^{T p}=T([1,2],[4,5],[3,3],[1,2],[1,3],[3,3],[1,2]) .
$$

We decide whether $\mathbf{A}^{T p}$ is universally robust.

Since $\tilde{A}=T(2,5,3,2,3,3,2), \tilde{A}$ fulfills condition $\mathscr{C}^{*}$ and by Theorem 5.1 $\mathbf{A}^{T p}$ fulfills condition $\mathscr{C}^{*}$. Since $h(\underline{A})=$ $h(\bar{A})=3$ and $\operatorname{per} A=1$, in view of Theorem 5.3 a given interval Toeplitz matrix is universally robust.

Theorem 5.3 represents a sufficient, but not necessary condition for the universal robustness of an interval Toeplitz matrix.

For each $k \in N^{+}, l \in N^{-}$we define the Toeplitz matrix $A^{(k l)}=\operatorname{Tp}\left(a_{-n+1}^{(k l)}, \ldots, a_{-1}^{(k l)}, a_{0}^{(k l)}, a_{1}^{(k l)}, \ldots, a_{n-1}^{(k l)}\right)$ as follows:

$a_{i}^{(k l)}= \begin{cases}\bar{a}_{i} & \text { for } i \in\{k, l\} \\ \underline{a}_{i} & \text { otherwise. }\end{cases}$

Theorem 5.4. Let $\mathbf{A}^{T p}$ be an interval Toeplitz matrix fulfilling condition $\mathscr{C}^{*} . \mathbf{A}^{T p}$ is universally robust if and only if $\underline{A}$ is robust and for each $k \in N^{+}, l \in N^{-}$such that $\min \left\{\bar{a}_{k}, \bar{a}_{l}\right\}>h(\underline{A})$ the matrix $A^{(k l)}$ is robust.

Proof. If $\underline{A}$ is not robust or there exist $k \in N^{+}, l \in N^{-}$such that $\min \left\{\bar{a}_{k}, \bar{a}_{l}\right\}>h(\underline{A})$ and the matrix $A^{(k l)}$ is not robust, then $\mathbf{A}^{T p}$ is not universally robust.

For the converse implication suppose that $\mathbf{A}^{T p}$ is not universally robust and $\underline{A}$ is robust. We will prove that there exist $k \in N^{+}, l \in N^{-}$such that $\min \left\{\bar{a}_{k}, \bar{a}_{l}\right\}>h(\underline{A})$ and the matrix $A^{(k l)}$ is not robust.

Let $A \in \mathbf{A}^{T p}$ be such that per $A \neq 1$, i.e., per $\mathscr{K}^{\prime} \neq 1$, where $\mathscr{K}^{\prime} \in \mathrm{SCC}^{\star} \mathscr{G}(A, h(A))$. Similarly as in the proof of Theorem 5.3 , the robustness of $\underline{A}$ implies $h(A)>h(\underline{A})$. Let $k \in N^{+}, l \in N^{-}$be such that $h^{+}(\bar{A})=a_{k}, h^{-}(A)=a_{l}$. Since $h^{+}\left(A^{(k l)}\right)=\bar{a}_{k}$ and $h^{-}\left(A^{(k l)}\right)=\bar{a}_{l}$ we obtain $h\left(A^{(k l)}\right)=$ $\min \left\{\bar{a}_{k}, \bar{a}_{l}\right\} \geq h(A)>h(\underline{A})$.

Let $j \in N^{*}$ be such that $j \notin I(A)$. Then $a_{j}^{(k l)}=\underline{a}_{j} \leq a_{j}<$ $h(A) \leq h\left(A^{(k l)}\right)$. Hence $j \notin I\left(A^{(k l)}\right)$. Thus $I\left(A^{(k l)}\right) \subseteq I(A)$. According to 2 , we obtain per $A \mid \operatorname{per} A^{(k l)}$ which implies $\operatorname{per} A^{(k l)} \neq 1$. Thus the matrix $A^{(k l)}$ is not robust.

According to Theorem 5.4 checking the universal robustness of a given interval Toeplitz matrix fulfilling condition $\mathscr{C}^{*}$ consists of $O(n)$ arithmetic operations needed for checking whether $\mathbf{A}^{T p}$ fulfills condition $\mathscr{C}^{*}, O(n)$ operations for checking the robustness of $\underline{A}$ by (2) and at most $n^{2} O(n)=O\left(n^{3}\right)$ operations for checking the robustness of matrices $A^{(k l)}$. So the complexity of checking the universal robustness of a given interval Toeplitz matrix fulfilling condition $\mathscr{C}^{*}$ is $O\left(n^{3}\right)$, which substantially improves the exponential algorithm for checking the universal robustness of an interval matrix in general case. 
Example 5.3. We decide whether $\mathbf{A}^{T p}$ is universally robust, if

$$
\mathbf{A}^{T p}=T([1,2],[4,5],[2,2],[1,2],[3,4],[3,5],[1,2]) .
$$

Since $\tilde{A}=T(2,5,2,2,3,3,2), \tilde{A}$ fulfills condition $\mathscr{C}^{*}$ and by Theorem 5.1 the given interval Toeplitz matrix $\mathbf{A}^{T p}$ fulfills condition $\mathscr{C}^{*}$. We have $h(\underline{A})=3$ and by (2) we get $\operatorname{per} \underline{A}=1$, so $\underline{A}$ is robust. Since $h(\bar{A})=5 \neq h(\underline{A})$, the sufficient condition from Theorem 5.3 is not satisfied, so we shall continue with checking the condition from Theorem 5.4.

For $k \in N^{+}$, the inequality $\bar{a}_{k}>3$ is fulfilled for $k \in$ $\{1,2\}$ and for $l \in N^{-}$, the inequality $\bar{a}_{l}>3$ is fulfilled for $l=-2$. We will construct the corresponding matrices $A^{(k l)}$ by (4).

For $k=1, l=-2$ we get $A^{(k l)}=T(1,5,2,1,4,3,1)$. We have $I\left(A^{(k l)}\right)=\{-2,1\}$ and by 2 we get per $A^{(k l)}=3$.

In view of Theorem 5.4 the given interval Toeplitz matrix is not universally robust.

\section{Example 5.4. Let}

$$
\mathbf{A}^{T p}=T([2,3],[6,6],[6,6],[1,2],[4,8],[3,4],[1,3]) .
$$

Decide whether $\mathbf{A}^{T p}$ is universally robust.

Since $\tilde{A}=T(3,6,6,2,4,4,3), \mathbf{A}^{T p}$ fulfills condition $\mathscr{C}^{*}$. We have $h(\underline{A})=4$ and by $(2)$ we get per $\underline{A}=1$, so $\underline{A}$ is robust. Since $h(\bar{A})=6 \neq h(\underline{A})$, we have to check the condition from Theorem 5.4

For $k \in N^{+}$, the inequality $\bar{a}_{k}>4$ is fulfilled for $k=1$ and for $l \in N^{-}$, the inequality $\bar{a}_{l}>4$ is fulfilled for $l \in$ $\{-2,-1\}$.

For $k=1, l=-2$ we get $A^{(k l)}=T(2,6,6,1,8,3,1)$. We have $I\left(A^{(k l)}\right)=\{-2,-1,1\}$. By (2) we get per $A^{(k l)}=1$.

For $k=1, l=-1$ we obtain $A^{(k l)}=T(2,6,6,1,8,3,1)$ which is identical to the matrix from the previous case, so $A^{(k l)}$ is robust.

According to Theorem 5.4 the given interval Toeplitz matrix is universally robust.

\section{REFERENCES}

[1] FIEDLER, M. - NEDOMA, J. - RAMÍK, J. - ROHN, J. - ZIMMERMANN, K.: Linear Optimization Problems with Inexact Data, Springer-Verlag, Berlin 2006.

[2] GAVALEC, M: Periods of special fuzzy matrices, Tatra Mountains Math. Publ. 16 (1999), 47-60.

[3] GAVALEC, M. - PLAVKA, J: Monotone interval eigenproblem in max-min algebra, Kybernetika 46 (3) (2010), 387-396.

[4] GAVALEC, M. - ZIMMERMANN, K: Classification of solutions to systems of two-sided equations with interval coefficients, Inter. J. of Pure and Applied Math. 45 (2008), 533-542.
[5] GAVALEC, M.: Computing matrix period in max-min algebra, Discrete Appl. Math. 75 (1997) 63-70.

[6] HORVATH, T. - VOJTÁŠ, P.: Induction of fuzzy and annotated logic programs, Inductive Logic Programming 4455 (2007) 260-274.

[7] MOLNÁROVÁ, M. - MYŠKOVÁ, H. PLAVKA, J.: The robustness of interval fuzzy matrices, Linear Alg. Appl. (2013), http://dx.doi.org/ 10.1016/j.laa.2012.12.020

[8] MYŠKOVÁ, H.: Interval systems of max-separable linear equations, Linear Alg. Appl. 403 (2005), 263272.

[9] MYŠKOVÁ, H.: Control solvability of interval systems of max-separable linear equations, Linear Alg. Appl. 416 (2006), 215-223.

[10] PLAVKA, J. - SZABÓ, P.: On the $\lambda$-robustness of matrices over fuzzy algebra, Discrete Applied Math. 159 Issue 5 (2011), 381-388.

[11] PLAVKA, J.: On the $O\left(n^{3}\right)$ algorithm for checking the strong robustness of interval fuzzy matrices, Discrete Applied Math. 160 (2012), 640-647.

[12] SANCHEZ, E.: Medical diagnosis and composite relations, In: M. M. Gupta, R. K. Ragade, R. R. Yager, Eds, Advances in Fuzzy Set Theory and Applications (North- Holland, Amsterdam- New York, 1979), 437444.

[13] TERANO, T. - TSUKAMOTO, Y.: Failure diagnosis by using fuzzy logic, In: Proc. IEEE Conference on Decision Control (New Orleans, LA, 1977), 13901395.

[14] ZADEH, I. A.: Toward a theory of fuzzy systems, In: R. E. Kalman, N. DeClaris, Eds., Aspects of Network and Systems Theory (Hold, Rinehart and Winston, New York, 1971), 209-245.

Received October 22, 2012, accepted December 23, 2012

\section{BIOGRAPHY}

Helena Myšková was born on 14. 1. 1964. She graduated (RNDr.) with distinction at the Faculty of Science at P. J. Šafárik University in Košice in 1987. She defended her $\mathrm{PhD}$ in the field of discrete mathematics in 2009; her thesis title was "Solving of systems and interval systems of linear equations over the max-plus and max-min algebra". She is working as a tutor at the Department of Mathematics and Theoretical Informatics since 1995. Her scientific research is focusing on interval computations in extremal algebras. 